\title{
Survivin-specific T-cell reactivity correlates with tumor response and patient survival: a phase-II peptide vaccination trial in metastatic melanoma
}

\author{
Jürgen C. Becker • Mads H. Andersen • Valeska Hofmeister-Müller • \\ Marion Wobser $\cdot$ Lidia Frey $\cdot$ Christiane Sandig $\cdot$ Steffen Walter • \\ Harpreet Singh-Jasuja $\cdot$ Eckhart Kämpgen $\cdot$ Andreas Opitz $\cdot$ Marc Zapatka $\cdot$ \\ Eva-B. Bröcker $\cdot$ Per thor Straten $\cdot$ David Schrama $\cdot$ Selma Ugurel
}

Received: 29 October 2011/ Accepted: 10 April 2012/Published online: 8 May 2012

(C) The Author(s) 2012. This article is published with open access at Springerlink.com

\begin{abstract}
Background Therapeutic vaccination directed to induce an anti-tumoral T-cell response is a field of extensive investigation in the treatment of melanoma. However, many vaccination trials in melanoma failed to demonstrate a correlation between the vaccine-specific immune response and therapy outcome. This has been mainly attributed to immune escape by antigen loss, rendering us in the need of new vaccination targets.

Patients and methods This phase-II trial investigated a peptide vaccination against survivin, an oncogenic inhibitor-of-apoptosis protein crucial for the survival of tumor cells, in HLA-A1/-A2/-B35-positive patients with
\end{abstract}

Electronic supplementary material The online version of this article (doi:10.1007/s00262-012-1266-9) contains supplementary material, which is available to authorized users.

J. C. Becker $(\varangle) \cdot$ D. Schrama · S. Ugurel

Department of Dermatology, Medical University Graz,

Auenbruggerplatz 8, $8010 \mathrm{Graz}$, Austria

e-mail: juergen.becker@medunigraz.at

S. Ugurel

e-mail: selma.ugurel-becker@medunigraz.at

M. H. Andersen · P. thor Straten

Center for Cancer Immune Therapy,

Department of Hematology,

Herlev University Hospital, Herlev, Denmark

V. Hofmeister-Müller · M. Wobser · L. Frey · C. Sandig ·

E.-B. Bröcker

Department of Dermatology,

Julius-Maximilians-University,

Würzburg, Germany

S. Walter · H. Singh-Jasuja

Immatics Biotechnologies $\mathrm{GmbH}$,

Tübingen, Germany treatment-refractory stage-IV metastatic melanoma. The study endpoints were survivin-specific T-cell reactivity (SSTR), safety, response, and survival (OS).

Results Sixty-one patients (ITT) received vaccination therapy using three different regimens. 55 patients (PP) were evaluable for response and survival, and 41/55 for SSTR. Patients achieving progression arrest $(\mathrm{CR}+\mathrm{PR}+$ SD) more often showed SSTRs than patients with disease progression $(p=0.0008)$. Patients presenting SSTRs revealed a prolonged OS (median 19.6 vs. 8.6 months; $p=0.0077)$; multivariate analysis demonstrated SSTR as an independent predictor of survival $(p=0.013)$. The induction of SSTRs was associated with gender (female vs. male; $p=0.014)$ and disease stage (M1a/b vs. M1c; $p=0.010$ ), but not with patient age, HLA type, performance status, or vaccination regimen.

\section{E. Kämpgen \\ Department of Dermatology, \\ University Hospital Erlangen, \\ Erlangen, Germany}

A. Opitz

Institute of Clinical Transfusion Medicine and Hemotherapy,

Julius-Maximilians-University, Würzburg, Germany

M. Zapatka

Division of Theoretical Bioinformatics,

German Cancer Research Center,

Heidelberg, Germany 
Conclusion Survivin-specific T-cell reactivities strongly correlate with tumor response and patient survival, indicating that vaccination with survivin-derived peptides is a promising treatment strategy in melanoma.

Keywords Melanoma - Survivin - T-cell reactivity · Therapy $\cdot$ Peptide vaccination

\section{Introduction}

Treatment for metastatic melanoma currently undergoes a transformation, changing the rigid scheme of dacarbazine as standard treatment in all stage-IV melanoma patients, attributed with a very low response rate and an extremely poor survival, into new, individualized therapeutic strategies. For the first time since decades, new drug therapies succeeded in demonstrating a significant survival benefit [1-3] in contrast to the numerous clinical trials reported before [4]. On the one hand, kinase inhibitors like the antiBRaf V600E agent vemurafenib clearly showed an improved survival in patients carrying the respective gene mutation [2]. On the other hand, the immunomodulating antibody ipilimumab, an enhancer of T-cell-mediated immune responses, also demonstrated a prolongation of survival in metastatic patients [3]. The latter agent is of particular interest, because it is supposed to generate persistent anti-tumoral immune responses and to hereby elicit long-term disease control and prolonged survival in the corresponding patients. Following these promising findings, T-cell-based treatment strategies, which mainly are active tumor-specific vaccinations, got again into the focus of clinical testing and evaluation in melanoma. The ultimate goal of these efforts would be to develop a therapeutic strategy consisting of a vaccination generating an efficient $\mathrm{T}$-cell response, which will thereafter be enhanced or at least maintained by non-specific immune modulation.

With regard to an active, antigen-specific immunotherapy, the identification of defined melanoma-associated antigens opened the opportunity to develop anti-melanoma vaccines [5]. In this respect, immunization with HLArestricted peptide epitopes derived from differentiation antigens is a strategy that has been vigorously pursued. Initial clinical trials using gp100 peptide vaccination plus IL-2 in stage-IV melanoma achieved objective responses in $12 / 32$ patients (42\%) [6]. Unfortunately, many of the thereafter studied vaccines aiming to induce immune responses against differentiation antigens failed to demonstrate clinical efficacy. Reviewing 440 patients, only four complete and nine partial responses were observed, rendering an objective response rate of $3 \%$ [7].

In the present study, we vaccinated melanoma patients not against a differentiation antigen, but against the oncogenic molecule survivin. Survivin is a bifunctional inhibitor-of-apoptosis protein that plays a key role in the protection of tumor cells from apoptosis. Accordingly, a potential down-regulation of survivin expression as a strategy of immune escape would severely impair a tumor cell's survival capacity. Moreover, survivin is overexpressed in melanoma, as well as in most cancer entities of epithelial and hematopoietic origin, and its overexpression is associated with disease progression and poor prognosis in the respective patients [8-10], which makes survivin an excellent candidate for therapeutic vaccinations against cancer [11, 12]. Preclinical studies using a survivin-specific DNA vaccine showed vaccine-induced immune responses eradicating pulmonary metastases in lung cancer patients [13]. Encouraged by these findings, we developed a peptide-based vaccine against survivin [14] and found this vaccine to induce $\mathrm{T}$-cell responses in heavily pretreated melanoma and pancreatic cancer patients without significant toxicity $[15,16]$. Furthermore, in situ peptide/HLAA2 multimer staining revealed infiltrating survivin-reactive $\mathrm{CD} 8+\mathrm{T}$ cells in soft tissue metastases of vaccinated patients. Driven by these promising results, the present phase-II study was intended to investigate the correlation between a vaccine-specific immune response and the corresponding treatment outcome. To improve the induction of survivin-specific immune responses, we twice amended the vaccination regimen. The first amendment (Regimen II) increased the frequency of vaccinations within the first 8 weeks, and the second amendment (Regimen III) introduced an upfront application of low-dose cyclophosphamide intended to deplete regulatory $\mathrm{T}$ cells.

\section{Patients and methods}

\section{Study design}

The primary endpoint of this single-arm, single-institution, prospective phase-II trial (NCT00108875; ClinicalTrials.gov) was a vaccine-specific immune response measured as ex vivo survivin-specific T-cell reactivity (SSTR). Secondary endpoints were safety, best overall response, overall survival (OS), and progression-free survival (PFS). The study endpoints were evaluated on intention-to-treat (ITT) and per-protocol (PP) basis. Patient recruitment was outlined as a total of 50 patients evaluable for response and survival. This sample size was calculated as sufficient for an exploratory analysis to draw correlations between vaccine-specific immune response and treatment outcome. The results of this analysis were intended to be implemented into the design of a currently planned randomized phase-III trial. 
Patient population

Patients with histologically confirmed metastatic melanoma were enrolled in accordance with the following main eligibility criteria: stage-IV disease following AJCC criteria [17]; at least one prior systemic therapy in stage-IV resulting in disease progression; at least one measurable target lesion according to RECIST [18]; stop of any previous anti-tumor or immunosuppressive treatment at least 4 weeks before the first vaccination; HLA type of A1 and/ or A2 and/or B35; overall performance status (OPS) according to ECOG criteria $\leq 2$; no active infection or autoimmune disease; and adequate bone marrow, hepatic, and renal functions. All types of metastatic sites were considered eligible including metastases to the brain, as well as all localizations of primary including cutaneous, mucosal, uveal, and unknown primaries. Prognostic factors of metastatic melanoma, serum lactate dehydrogenase (LDH), as well as OPS, were recorded at treatment onset. The study protocol was approved by the Institutional Review Board, and written informed consent was signed by all patients prior to enrollment.

\section{Vaccination therapy}

Patients received vaccinations with HLA-restricted peptide epitopes derived from survivin [14]. The peptide sequences were modified in order to enhance their HLA binding affinity $[19,20]$. The peptides used were FTELTLGEF (HLA-A1; PolyPeptide Laboratories, Wolfenbüttel, Germany), LMLGEFLKL (HLA-A2; Clinalfa, Sissach, Switzerland), and EPDLAQCFY (HLA-B35; PolyPeptide Laboratories), all of pharmaceutical (GMP) quality. Each vaccination comprised $100 \mu \mathrm{g}$ of each peptide matching the patient's HLA type emulsified in $1 \mathrm{ml}$ Montanide $^{\circledR}$ ISA-51 (Seppic, Paris, France) and was administered by deep subcutaneous injections. Three different vaccination regimens were used in consecutive order: vaccinations in weeks 1, 2, and 5, followed by 4-week intervals (Regimen I); weekly vaccinations in week $1-8$, followed by 4 -week intervals (Regimen II); and the schedule of Regimen II preceded by a single i.v. dose of cyclophosphamide $250 \mathrm{mg} / \mathrm{m}^{2} 24 \mathrm{~h}$ prior to the first vaccination (Regimen III). Toxicity was evaluated using common toxicity criteria (CTC) 2.0 (http://ctep.cancer.gov/reporting/ctc.html).

Ex vivo detection of survivin-specific T-cell reactivity

Enzyme-linked immunospot (ELISPOT) assays were used to quantify IFN $\gamma$-releasing survivin-specific effector T cells in samples of peripheral blood mononuclear cells (PBMCs) as described previously [21]. Briefly, nitrocellulose-bottomed 96-well plates (MultiScreen MAIP N45, Millipore,
Schwalbach, Germany) were coated with an anti-IFN $\gamma$ antibody (1-D1K, Mabtech, Stockholm, Sweden), and nonspecific binding was blocked using AIM-V (Life Technologies, Gaithersburg, MD). Lymphocytes were isolated from heparinized peripheral blood samples of study patients and subsequently incubated overnight at $37^{\circ} \mathrm{C}$ at different cell concentrations together with the respective HLA-matched survivin epitope-specific peptides and T2 cells. The peptides used in the assay were the same as those used for patient vaccination. After two washing procedures, the biotinylated detection antibody (7-B6-1-Biotin, Mabtech) was added. Specific binding was visualized using alkaline phosphatase-avidin together with the respective substrate (Life Technologies). The reaction was stopped on the appearance of dark purple spots as a measure of IFN $\gamma$-release, which was quantified using the AlphaImager System (Alpha Innotech, San Leandro, CA). Reactivity was considered positive, if the IFN $\gamma$ release of cells incubated with a specific peptide was more than tripling the release of the same cells incubated without a peptide in at least two independent experiments.

\section{MHC multimer assay}

Peptides for HLA-class-I multimers were ILKEPVHGV from HIV-1-RT-476-484, LTLGEFLKL from human parental survivin 96-104, and its modified form LMLGEFLKL. Biotinylated recombinant peptide-HLA$\mathrm{A}^{*}$ 0201-monomers and multimers were produced as previously described [22]. Dual MHC multimer assessment was performed 12 days after a single round of in vitro sensitization as described previously [23]. Briefly, PBMCs were pulsed with $10 \mu \mathrm{g} / \mathrm{ml}$ readout class-I peptides for $2 \mathrm{~h}$, then pelleted, resuspended, and cultured for 13 days in $\mathrm{X}$-vivo 15 (Lonza, Verviers, Belgium) plus $10 \%$ heatinactivated human $\mathrm{AB}$ serum (C.C.Pro, Neustadt, Germany), $2 \mathrm{mM} \mathrm{L-glutamine} \mathrm{(Lonza),} \mathrm{and} 40 \mathrm{U} / \mathrm{ml} \mathrm{IL}-2$ (Novartis, Munich, Germany). Harvested PBMCs were stained first with Live/Dead Aqua (Invitrogen, Karlsruhe, Germany), multimer-PE, and multimer-APC (each at 5

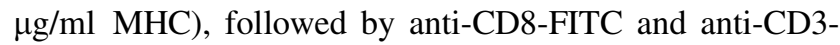
PacificBlue (Becton-Dickinson, Heidelberg, Germany). Cells were fixed and analyzed on a LSRII cytometer (BectonDickinson), gated on live CD8+ CD3+ lymphocytes.

Assessment of tumor response and survival

Patients who completed at least 28 days of vaccination, corresponding to two vaccinations in Regimen I and four vaccinations in Regimens II and III, respectively, were considered evaluable for treatment response and survival (PP). Tumor response was assessed by $\mathrm{CT}$ and/or MRI imaging in 8-week intervals and evaluated according to 
Table 1 Patient characteristics at enrollment, treatment efficacy, and outcome

\begin{tabular}{|c|c|c|}
\hline & ITT $61(100.0 \%)$ & PP $55(100.0 \%)$ \\
\hline \multicolumn{3}{|l|}{ Gender } \\
\hline Male & $39(63.9 \%)$ & $35(63.6 \%)$ \\
\hline Female & $22(36.1 \%)$ & $20(36.4 \%)$ \\
\hline Median age/years (range) & $62.5(28.4-82.7)$ & $61.3(28.4-82.7)$ \\
\hline \multicolumn{3}{|l|}{ HLA type $^{\mathrm{a}}$} \\
\hline A1 & $20(32.8 \%)$ & $19(34.5 \%)$ \\
\hline $\mathrm{A} 2$ & $42(68.9 \%)$ & $32(58.2 \%)$ \\
\hline B35 & $15(24.6 \%)$ & $15(27.3 \%)$ \\
\hline \multicolumn{3}{|l|}{ Serum LDH } \\
\hline$\leq \mathrm{UNL}$ & $38(62.3 \%)$ & $37(67.3 \%)$ \\
\hline$>\mathrm{UNL}$ & $23(37.7 \%)$ & $18(32.7 \%)$ \\
\hline \multicolumn{3}{|l|}{ Performance status (ECOG) } \\
\hline 0 & $45(73.8 \%)$ & $44(80.0 \%)$ \\
\hline 1 & $12(19.7 \%)$ & $10(18.2 \%)$ \\
\hline 2 & $4(6.5 \%)$ & $1(1.8 \%)$ \\
\hline \multicolumn{3}{|l|}{ M category (AJCC) } \\
\hline M1a & $6(9.8 \%)$ & $6(10.9 \%)$ \\
\hline M1b & $9(14.8 \%)$ & $9(16.4 \%)$ \\
\hline M1c & $46(75.4 \%)$ & $40(72.7 \%)$ \\
\hline \multicolumn{3}{|l|}{ Inflammatory reaction at vaccination sites } \\
\hline Yes & $18(29.5 \%)$ & $18(32.7 \%)$ \\
\hline No & $43(70.5 \%)$ & $37(67.3 \%)$ \\
\hline \multicolumn{3}{|l|}{ Survivin-specific T-cell reactivity $(\mathrm{SSTR})^{\mathrm{b}}$} \\
\hline Positive & $13(21.3 \%)$ & $13(23.6 \%)$ \\
\hline Negative & $31(50.8 \%)$ & $28(50.9 \%)$ \\
\hline Not assessed & $17(27.9 \%)$ & $14(25.5 \%)$ \\
\hline \multicolumn{3}{|l|}{ Best overall response $^{c}$} \\
\hline CR & $1(1.6 \%)$ & $1(1.8 \%)$ \\
\hline PR & $3(4.9 \%)$ & $3(5.5 \%)$ \\
\hline SD & $7(11.5 \%)$ & $7(12.7 \%)$ \\
\hline $\mathrm{PD}$ & $50(82.0 \%)$ & $44(80.0 \%)$ \\
\hline Objective response $(\mathrm{CR}+\mathrm{PR})$ & $4(6.6 \%)$ & $4(7.3 \%)$ \\
\hline Progression arrest $(\mathrm{CR}+\mathrm{PR}+\mathrm{SD})$ & $11(18.0 \%)$ & $11(20.0 \%)$ \\
\hline Median progression-free survival months $(95 \% \mathrm{CI})^{\mathrm{d}}$ & $2.8(2.2-3.9)$ & $3.0(2.4-4.1)$ \\
\hline Median overall survival months $(95 \% \mathrm{CI})^{\mathrm{d}}$ & $9.1(6.1-11.3)$ & $9.8(6.4-11.9)$ \\
\hline
\end{tabular}

$I T T$ intention-to-treat, $P P$ per-protocol, $L D H$ lactate dehydrogenase, $U N L$ upper normal limit, ECOG Eastern Cooperative Oncology Group, $A J C C$ American Joint Committee on Cancer, $C R$ complete response, $P R$ partial response, $S D$ stable disease, $P D$ progressive disease, $C I$ confidence interval

${ }^{\text {a }}$ Multiple entries possible

b Survivin-specific T-cell reactivities (SSTR) were quantified by ELISPOT as described in "Patients and methods" and classified as positive or negative as described in "Results"

${ }^{c}$ Best overall response was defined as the best tumor response recorded from the start of treatment until removal of the patient from the trial

${ }^{\mathrm{d}}$ Survival was measured from the date of first vaccination until the date of death or disease progression, respectively

RECIST [18]. Complete (CR) and partial (PR) responses were combined as objective response (OR). Patients who died from melanoma rapidly after treatment onset were considered as progressive disease (PD). Best overall response was defined as the best response recorded between the start and the end of treatment; best overall responses of stable disease (SD) or better were considered as progression arrest $(\mathrm{CR}+\mathrm{PR}+\mathrm{SD})$ [18]. All CT and 
MRI scans from patients showing progression arrest were retrospectively reviewed by an independent radiologist. OS and PFS were measured from the date of first vaccination until the date of death or disease progression, respectively. If no such event occurred, the date of the last patient contact was used as endpoint of survival assessment (censored observation).

\section{Statistical analysis}

Fisher's exact test was used to compare T-cell reactivities, tumor response rates, and toxicities between groups. Survival curves and median survival times were calculated using the Kaplan-Meier method for censored failure time data. The logrank test was used for comparison of survival probabilities between groups. $95 \%$ confidence intervals for median survival were calculated using the method of Brookmeyer [24]. Multivariate testing using the proportional hazards model of Cox was applied to test for independent predictors of survival in adjustment with the clinical covariates age, gender, and disease stage (M category). All $p$ values are two-tailed and unadjusted for potential multiple comparisons to allow a hypothesisbuilding exploratory data analysis; $p<0.05$ was considered statistically significant.

\section{Results}

Patient characteristics and study flow

Between 03/2003 and 11/2007, 61 patients were enrolled into the study (ITT); detailed patient characteristics are presented in Tables 1 and S1 (S, supplementary; all supplementary materials available online); the distribution on the different vaccination regimens can be seen in Fig. 1 and Table S1. All 61 patients met the eligibility criteria and started vaccination therapy within 1 week following enrollment. 6/61 patients $(9.8 \%)$ had to be excluded from PP analysis due to less than 28 days on treatment (Fig. 1); $55 / 61$ patients $(90.2 \%)$ were evaluable for treatment response and survival (PP).

\section{Survivin-specific T-cell reactivity (SSTR)}

41/55 PP patients (74.5\%; Regimen I = 24 pts; Regimen $\mathrm{II}=10$ pts; Regimen III = 7 pts) consented in peripheral blood withdrawal and analysis of PBMCs by ex vivo ELISPOT for SSTRs before the first vaccination (at baseline) and every 8 weeks thereafter until termination of study treatment. Patients demonstrating a positive ex vivo detection of SSTRs at at least one time point during the first 16 weeks of ongoing vaccination (either at baseline and/or at week 8 and 16, respectively) were defined "positive"; patients without positive reactivity were considered "negative." $13 / 41$ patients $(31.7 \%)$ presented positive SSTRs during vaccination. These reactivities in the majority of patients were first detected at 8 weeks following the first vaccination and stayed positive for up to $60+$ months; two of the 13 patients (15.4\%) showing positive SSTRs were already positive at baseline and stayed positive during ongoing vaccination. The presence of SSTRs was neither influenced by the vaccination regimen ( $p=0.96$; Fig. 2a) nor by the patients' HLA type ( $p=0.73$; Fig. $2 b$ ). Interestingly, female patients presented SSTRs significantly more often than males ( $p=0.014$; Fig. $2 c$ ). Patients in stages M1a/b more often revealed SSTRs than patients in stage M1c ( $p=0.010$; Fig. 2d); moreover, a trend toward less frequent SSTRs was observed in patients with elevated serum LDH compared to patients with normal LDH levels ( $p=0.16$; data not shown). Patients with uveal melanoma also showed a trend toward less frequent SSTRs compared to patients with melanomas of other origins ( $p=0.056$; data not shown). Patients' OPS $(p=0.57)$ and age at therapy onset $(p=0.41)$ had no significant impact on SSTRs (data not shown).

\section{MHC multimer staining}

Flow cytometry analysis using soluble survivin peptideMHC multimers, which specifically interact with respective T-cell receptors, were performed in exemplary patients who showed positive SSTRs at 2 months after onset of vaccination. Comparison of the results obtained from the use of HLA multimers, which braced the modified or the wild-type survivin epitopes, respectively, revealed that $\mathrm{T}$ cells reactive against either multimer could be detected among the PBMCs of vaccinated patients (Fig. 3).

Tumor response and patient survival

The database was frozen in December 2008 with a median follow-up time of 45 months. Tumor response to treatment is presented in Table 1; no significant differences could be observed between the three treatment regimens. The characteristics of patients showing a progression arrest are given in Table 2. Considering the PP population, 49 deaths occurred, and six patients were still alive with four of them receiving ongoing vaccination. A detailed presentation of OS and PFS is provided in Table 1 . With regard to known prognostic factors of metastatic melanoma, we observed a favorable OS in patients with normal versus elevated serum LDH ( $p=0.0009$; Figure S1A), in patients at stage M1a/b versus M1c $(p=0.0074$; Figure S1B), and in patients presenting an OPS $=0$ versus OPS $>0(p=0.21$; Figure $\mathrm{S} 1 \mathrm{C})$. Neither vaccination regimen nor patients' gender or 
Fig. 1 Schematic presentation of the study flow (CONSORT diagram). ITT intention-to-treat, $P P$ per-protocol

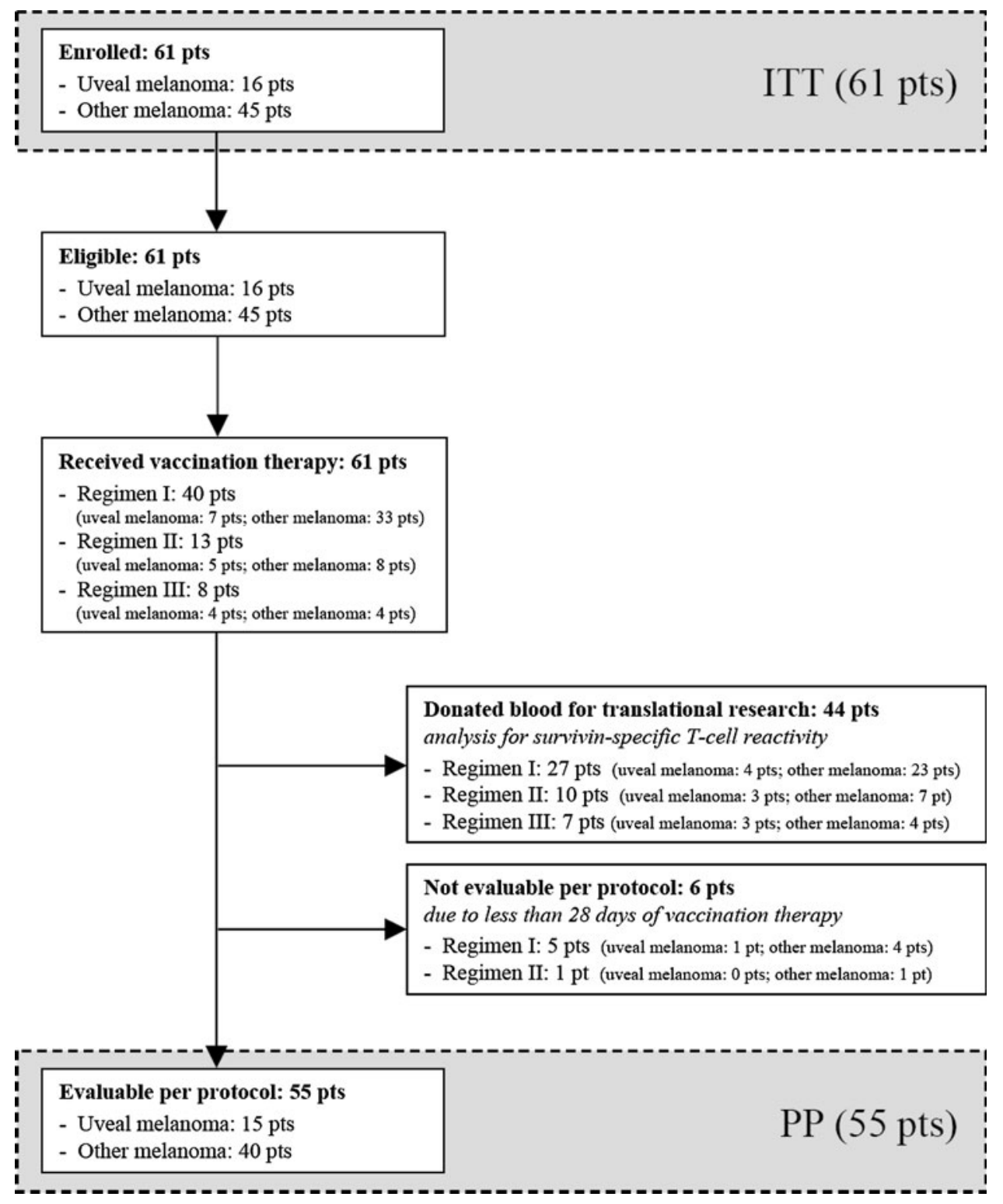

HLA type had a significant impact on overall survival (data not shown). Patients with uveal melanoma revealed an impaired survival compared to patients with melanoma originating from other localizations $(p=0.039$; Figure S1D).

Survivin-specific T-cell reactivity correlates with tumor response and patient survival

Concerning tumor response, patients experiencing a progression arrest $(\mathrm{CR}+\mathrm{PR}+\mathrm{SD})$ under vaccination revealed significantly more often SSTRs than patients with a disease progression ( $p=0.0008$; Fig. 2e). Moreover, patients presenting a SSTR during vaccination revealed a significantly prolonged OS compared to patients showing no survivin-specific reactivity (median 19.6 vs. 8.6 months, $p=0.0077$; Fig. 4a). Multivariate analysis using the proportional hazards model of Cox including the parameters age, gender, disease stage ( $M$ category), and SSTR revealed SSTR $(p=0.013)$ and disease stage $(p=0.027)$ as independent prognostic predictors. Age $(p=0.38)$ and gender $(p=0.12)$ resulted as no independently significant prognostic parameters.

\section{Treatment-related toxicity}

The majority of treatment-related side effects were mild to moderate (CTC grade 1-2). The most common toxicities were fever and chills on the day of vaccination and inflammatory reactions at the injection sites characterized by erythematous, dense, and painful nodules arising in about $30 \%$ of patients (Table 1; Fig. 5). Interestingly, the 
Fig. 2 Survivin-specific T-cell reactivities (SSTR) of the per-protocol population ( 55 patients) as detected by ELISPOT, diagramed by a vaccination regimens; b patients' HLA type; c patients' gender; $\mathbf{d}$ M category according to AJCC criteria; e best overall response grouped as progression arrest

$(\mathrm{CR}+\mathrm{PR}+\mathrm{SD})$ and progression $(\mathrm{PD})$; and f inflammatory reaction at the vaccination sites. Patients demonstrating a positive detection of SSTR at at least one time point during the first 16 weeks of ongoing vaccination were defined positive (green bars); patients without this reactivity were considered negative (red bars). Fisher's exact test was used to compare T-cell reactivities between groups; $p$ values are provided above the corresponding bars
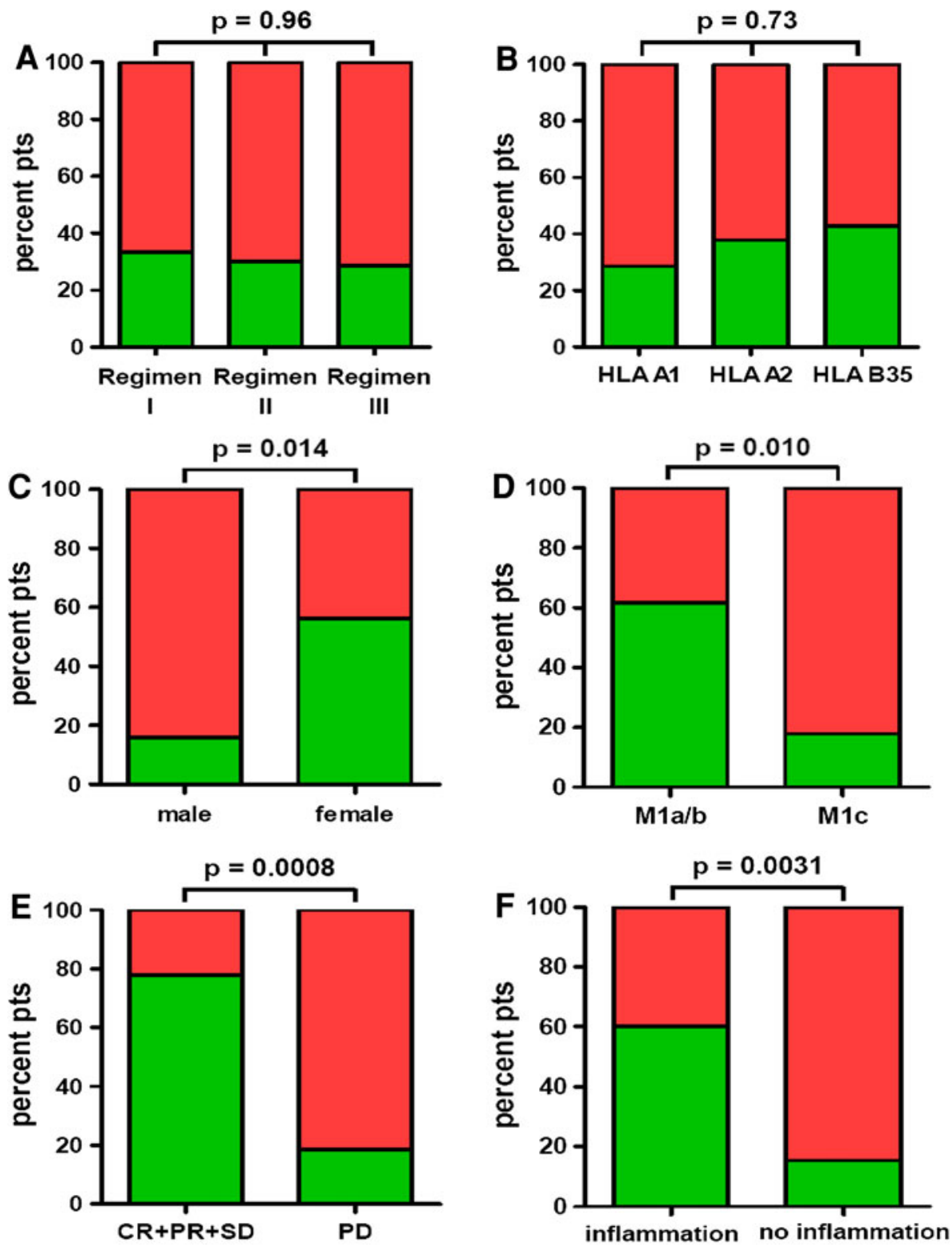

survivin-specific T-cell reactivity positive survivin-specific $\mathrm{T}$-cell reactivity negative occurrence of these post-vaccination inflammatory reactions was strongly associated with the presence of SSTRs ( $p=0.0031$; Fig. 2f). Moreover, patients presenting vaccination-induced inflammatory reactions showed a trend toward a favorable survival ( $p=0.13$; Fig. $4 \mathrm{~b})$. CTC grade 3-4 toxicities potentially related to study therapy are summarized in Table S2. Most of these toxicities were unspecific conditions, which must be considered rather tumor-related than therapy-related. None of the observed toxicities required any action, and no significant differences could be observed between the three vaccination regimens tested (data not shown).

\section{Discussion}

Encouraged by our first promising observation of a successful survivin peptide vaccination in heavily pretreated stage-IV melanoma patients [15], we tested its safety, immunogenicity, and clinical efficacy in the present phaseII trial. Hereby, the major goal was to show a correlation between survivin-specific immune response and treatment outcome.

Sixty-one patients (ITT) were included into this trial; 55 (PP) were evaluable for treatment response and survival, and 41/55 were evaluable for SSTR. Notably, for all 
A

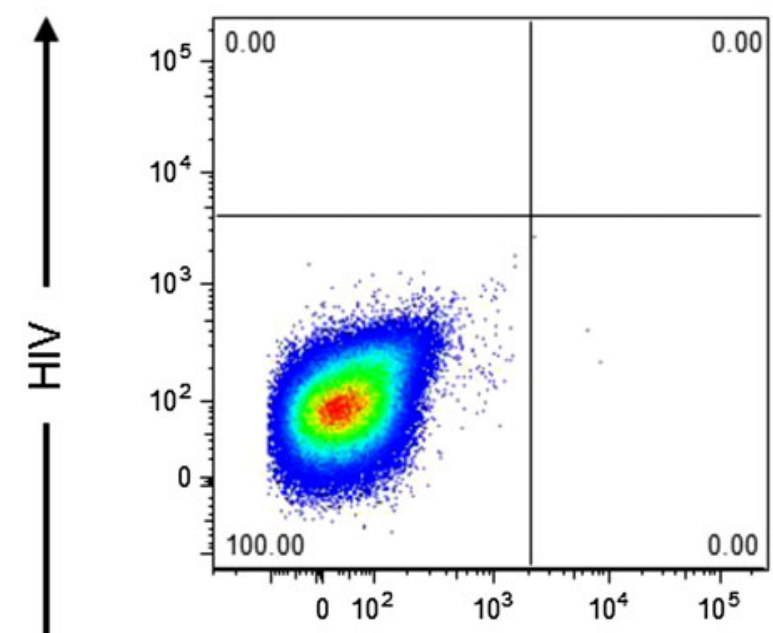

Post-Vaccination

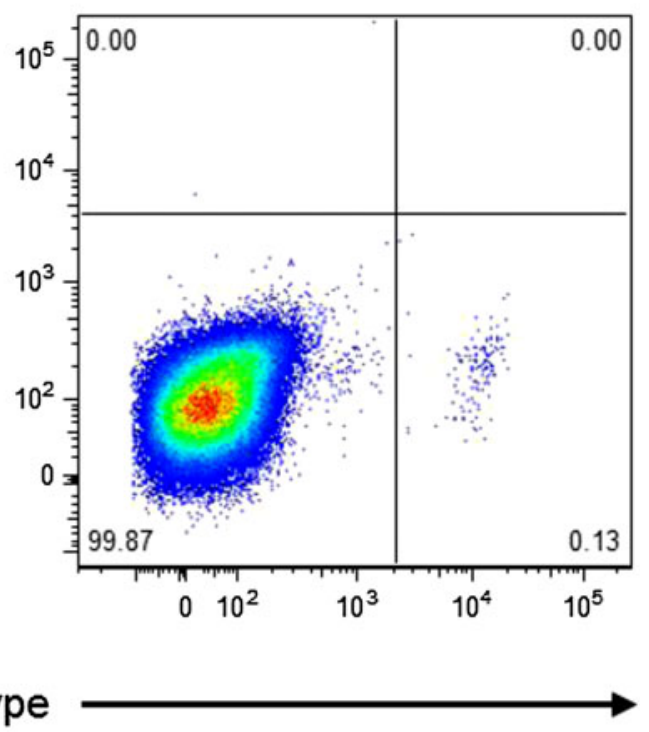

B
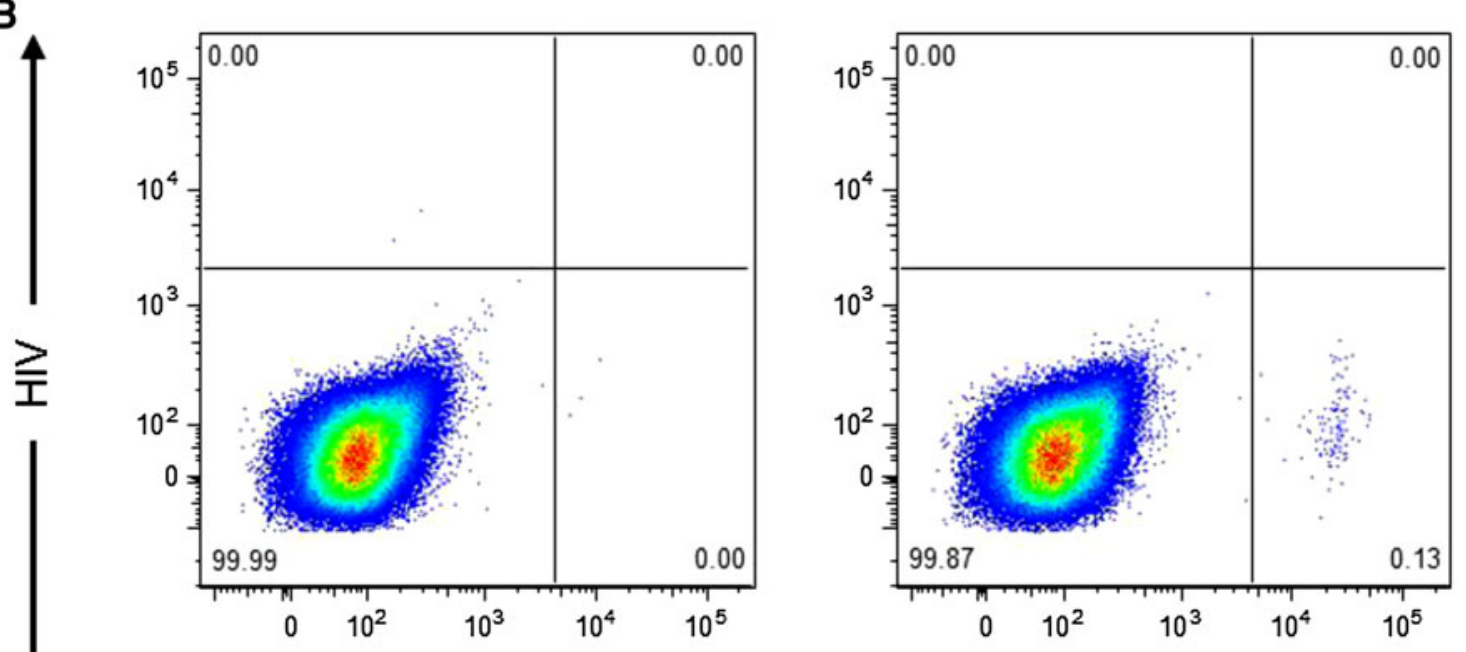

\section{modified}

Fig. 3 Vaccination-induced CD8 $+\mathrm{T}$ cells recognize the modified and wild-type HLA-A2-restricted survivin epitopes. PBMCs drawn from a HLA-A2+ patient before (left panels) and after 8 weeks (right panels) of vaccination in Regimen I were incubated with the modified survivin peptide LMLGEFLKL, the wild-type survivin peptide LTLGEFLKL, and the HIV-derived peptide ILKEPVHGV as

patients, disease progression under the previous treatment line was confirmed by imaging studies. With $>70 \%$ of the PP population in stage M1c, $>50 \%$ harboring two or more metastatic sites, $>35 \%$ already received two or more therapies, and $15 \%$ presenting brain metastases, the patient cohort was characterized by an extremely poor prognosis. Nevertheless, four patients $(7 \%)$ achieved an OR, and seven patients ( $13 \%$ ) a SD; thus, $20 \%$ revealed a progression arrest translating into a median OS of negative control. Cells were stained with the HLA multimers HIV (A*0201-ILKEPVHGV) and wild-type survivin (A*0201LTLGEFLKL) (a) or with HLA multimers HIV and modified survivin (A*0201-LMLGEFLKL) (b). Cells were analyzed by flow cytometry and gated on live CD8+ CD3+ lymphocytes

31.4 months. The established prognostic factors of advanced melanoma, M category, OPS, and localization of the primary, showed a significant impact on overall survival, whereas HLA type and vaccination regimen did not. Notably, similar factors, that is, $\mathrm{M}$ category and localization of the primary, revealed an impact on the presence of SSTRs, whereas again HLA type and vaccination regimen did not. SSTRs were significantly more often observed in women. Notably, female patients have been reported to 


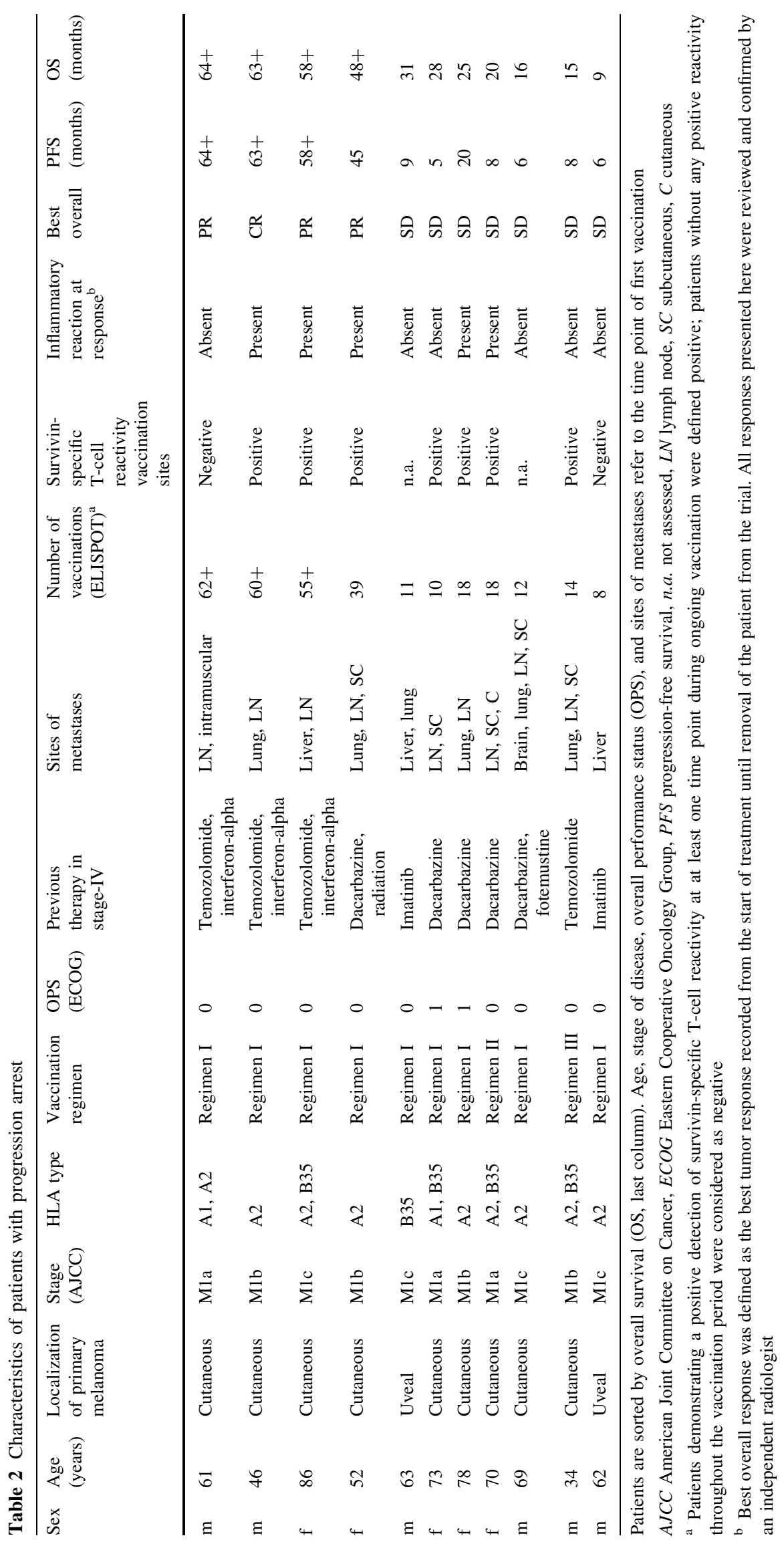



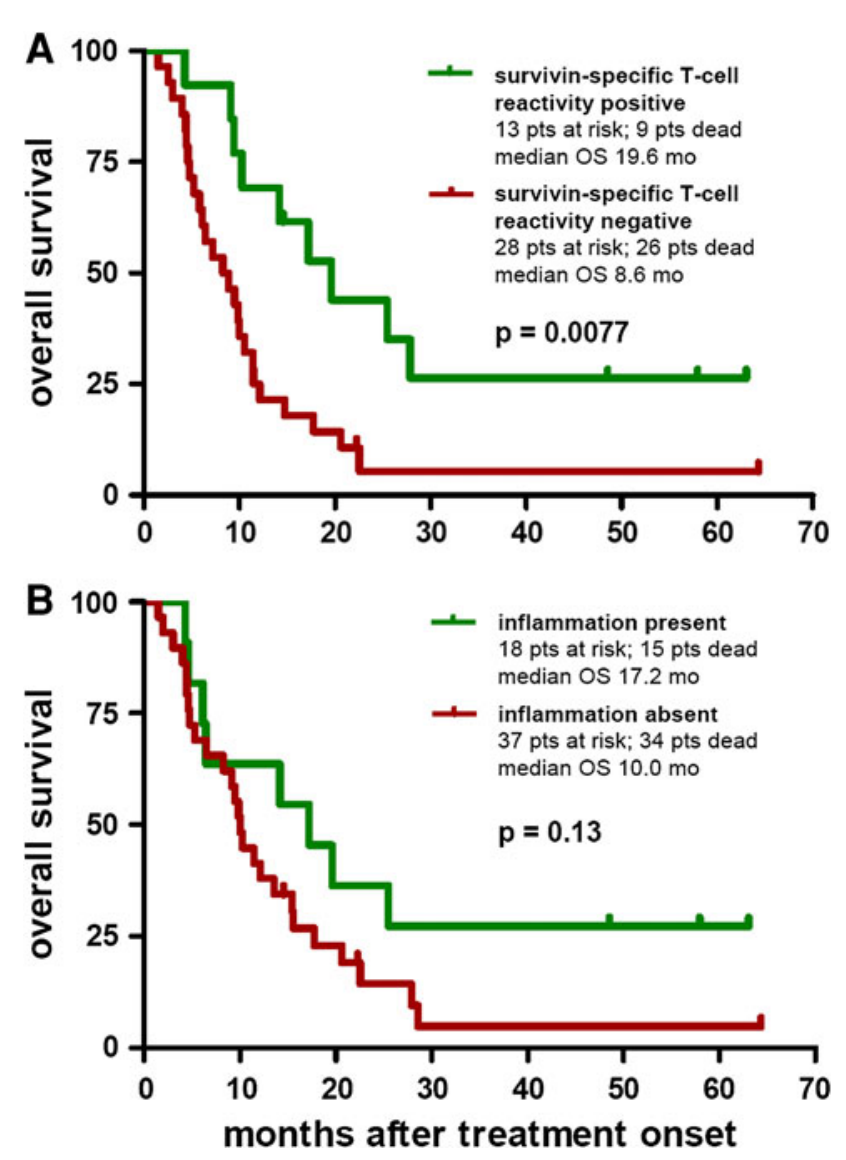

Fig. 4 Kaplan-Meier plots depicting the probability of overall survival (OS) of the per-protocol population (55 patients) by a survivin-specific T-cell reactivity (SSTR) detected by ELISPOT as described in "Patients and methods" and classified as positive or negative as described in "Results"; and $\mathbf{b}$ inflammatory reaction at the vaccination sites. Differences between groups were calculated using the logrank test; $p$ values are provided within the corresponding plots. Censored observations are indicated by vertical bars

show better responses to anti-melanoma immunotherapies [25]; however, this subject has not yet been studied in detail. This phenomenon might be explained by the stronger immune and autoimmune reactivities observed in women compared to men, linked to the wide repertoire of immune-related genes on the $X$ chromosome [26]. Most importantly, the present study shows a strong correlation between the rise of a specific $\mathrm{T}$-cell response against survivin during vaccination and therapy outcome in terms of tumor response $(p=0.0008)$ and overall survival ( $p=0.0077$, with SSTRs being an independent predictor of patients' survival. Interestingly, we observed an association between the presence of SSTRs and the occurrence of inflammatory reactions at the injection sites. Indeed, patients presenting these inflammatory reactions showed a trend toward a favorable survival. This observation has to be further investigated in future trials, but, nevertheless, suggests that the onset of inflammatory reactions visible at the cutaneous vaccination sites of patients treated with survivin-specific peptides might be used as an easily accessible surrogate marker for a survivin-specific T-cell response to vaccination.

Explanations are needed for the frequently reported lack of correlation between vaccine-specific $\mathrm{T}$-cell responses and the clinical outcome of vaccination trials. One critical point is the immunomonitoring of vaccinated patients. To date, there is no consensus on the required assays, and standard operating procedures are missing [27]. This problem has severely limited the ability to compare the results of different vaccination trials [28]. In the present study, SSTRs were analyzed by ex vivo ELISPOT assays of peripheral blood samples of 41 vaccinated patients who consented to donate blood, revealing that $31.7 \%$ of the analyzed patients presented a robust and reproducibly detectable survivin-specific immune response. We chose the ex vivo ELISPOT assay as the main readout due to our previous observation of (1) higher frequencies of SSTRs after in vitro stimulation, but (2) lower reproducibility, and (3) much lower correlation of the detected reactivities with the patients' clinical course, indicating that results obtained from in vitro stimulated assays, at least in our hands, may be more difficult to interpret.

Another explanation for the lack of correlation between vaccine-specific T-cell reactivities and patients' clinical outcome may be the choice of the target antigens [12, 14]. Tumor cell escape from immune response can be acquired by several mechanisms, with antigen loss as one of the most important ones. Unfortunately, melanocytic differentiation antigens, against which vaccination trials in melanoma have been most vigorously pursued, are ranking among this category [7]. In contrast, survivin expression is directly associated with the oncogenic phenotype of tumor cells, which ensures its maintained expression even under immuno-selective pressure [8-10, 12, 14].

We used peptides that were modified in one amino acid compared to the original epitopes in order to enhance HLA binding affinity. It has been recently suggested that vaccination with affinity-improved peptide epitopes gives rise to immune responses against the modified epitope only, but not against the wild type [29]. However, our results from epitope-MHC multimer staining in exemplary patients demonstrate that vaccination with affinity-improved survivin peptides induced $\mathrm{T}$-cell responses against both, the modified as well as the native peptide.

In conclusion, the results of the present trial not only demonstrate the clinical activity of a survivin-based peptide vaccination but also show a strong correlation between the presence of anti-survivin $\mathrm{T}$-cell responses and an improved clinical course of the disease as documented by progression arrest and overall survival. Moreover, survivinspecific T-cell reactivities could be shown as an independent predictor of survival in vaccinated patients. This 
Fig. 5 Vaccination sites in two representative patients showing a weak (a-d) and a strong $(\mathbf{e}-\mathbf{h})$ inflammatory reaction, respectively. Staining with hematoxylin and eosin.

Magnification $\times 10(\mathbf{b}, \mathbf{f}), \times 20$

$(\mathbf{c}, \mathbf{g})$, and $\times 40(\mathbf{d}, \mathbf{h})$
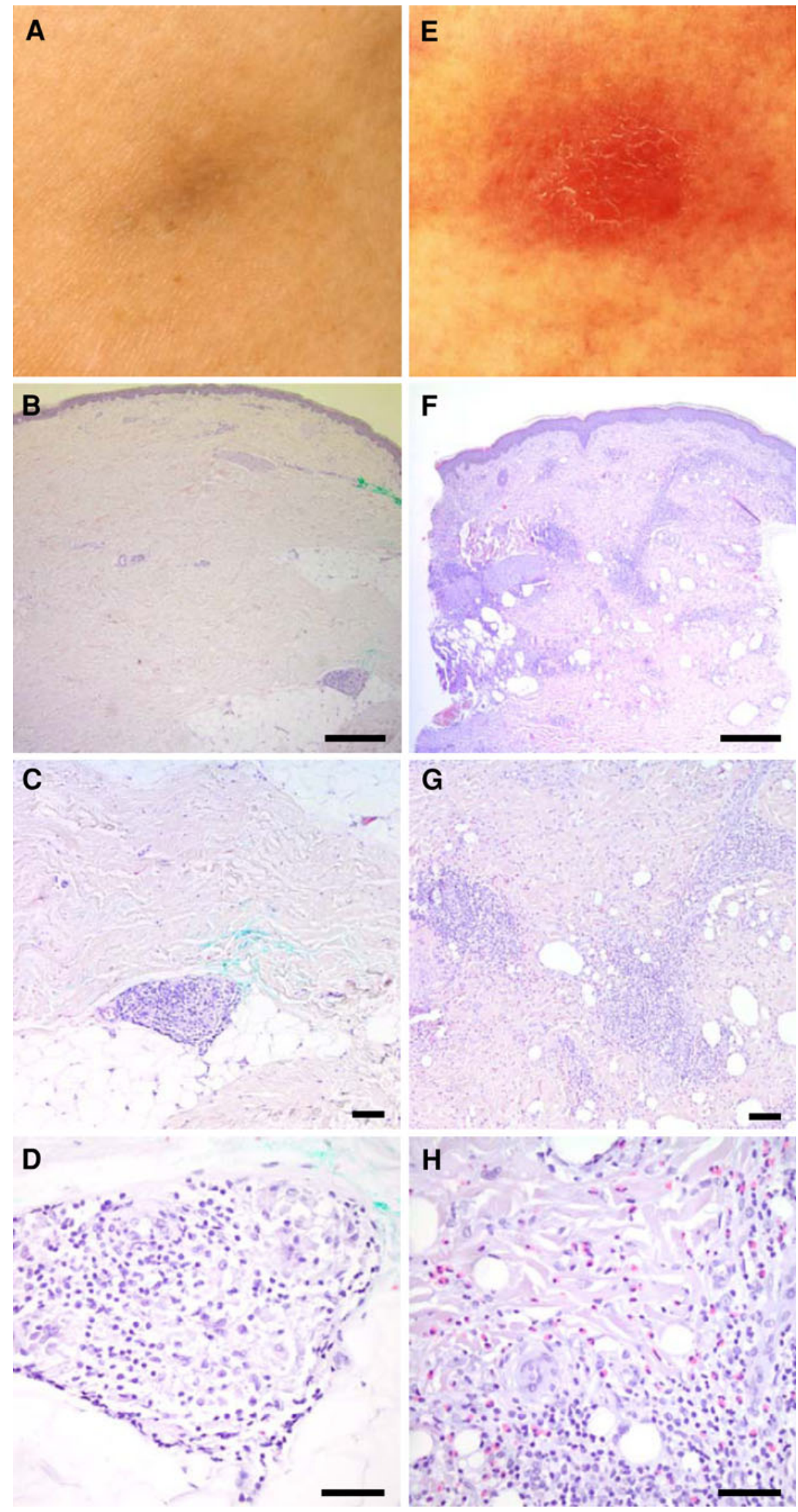

G
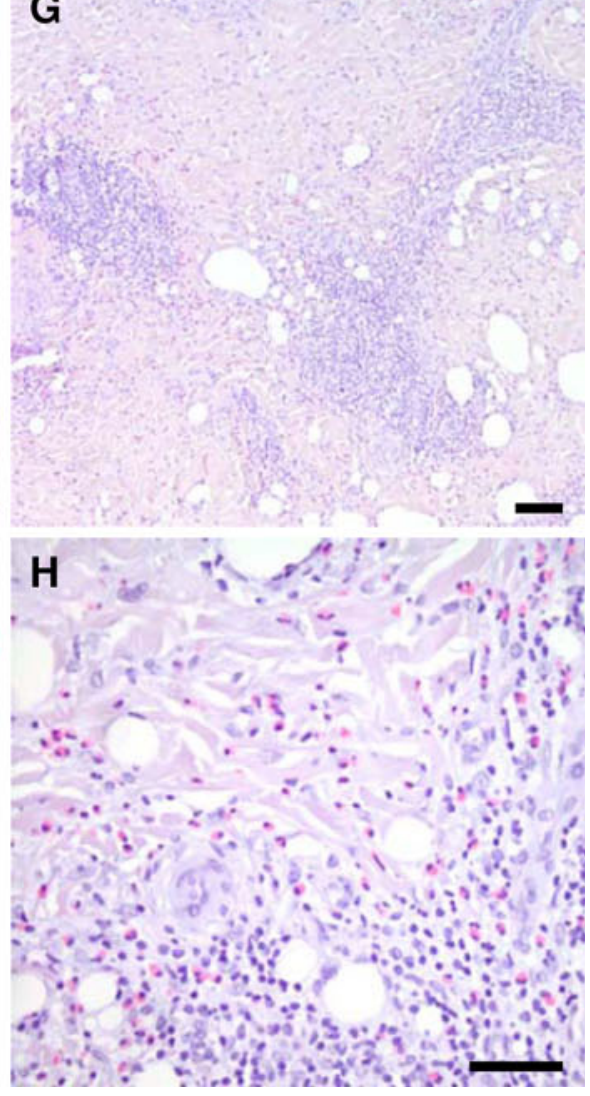
implies that the antigen-specific T-cell reactivity (SSTR) detectable ex vivo from the patients' blood material within the first months after onset of vaccination could be used as a surrogate marker of therapy outcome in terms of tumor response and overall survival. Thus, the attractiveness of survivin as an universal tumor antigen with oncogenic function could be translated into clinical activity in therapy-refractory, advanced melanoma patients. A survivinspecific peptide vaccination elicits an ex vivo measurable T-cell response, which renders this treatment as suitable to be applied before or together with an enhancer of T-cell response, for example, ipilimumab. Clinical trials are needed to further investigate this treatment approach.

Acknowledgments We like to express our appreciation to all the patients participating in this clinical trial and in the associated translational research. Furthermore, we like to thank the medical and technical staff who helped to conduct this trial. This work was supported by the Deutsche Forschungsgemeinschaft (grant KFO124) and an educational grant of Merck Serono.

Conflict of interest Jürgen C. Becker: advisory boards/speakers bureau (BMS, Cephalon, GSK, Merck Serono, Novartis, Roche); Mads H. Andersen: share holder (RhoVac); Valeska HofmeisterMüller: none; Marion Wobser: none; Lidia Frey: none; Christiane Sandig: none; Steffen Walter: employee, co-inventor, share options (immatics); Harpreet Singh-Jasuja: employee, co-inventor, share options, share holder (immatics); Eckhart Kämpgen: speakers bureau (BMS, Merck Serono, MSD, Novartis, Roche); Andreas Opitz: none; Marc Zapatka: none; Eva-B. Bröcker: none; Per thor Straten: advisory board (Dendreon corporation); David Schrama: speakers bureau (BMS); Selma Ugurel: speakers bureau (Amgen, BMS, Novartis, Roche).

Open Access This article is distributed under the terms of the Creative Commons Attribution License which permits any use, distribution, and reproduction in any medium, provided the original author(s) and the source are credited.

\section{References}

1. Schwartzentruber DJ, Lawson DH, Richards JM, Conry RM, Miller DM, Treisman J, Gailani F, Riley L, Conlon K, Pockaj B, Kendra KL, White RL, Gonzalez R, Kuzel TM, Curti B, Leming PD, Whitman ED, Balkissoon J, Reintgen DS, Kaufman H, Marincola FM, Merino MJ, Rosenberg SA, Choyke P, Vena D, Hwu P (2011) gp100 peptide vaccine and interleukin-2 in patients with advanced melanoma. N Engl J Med 364:2119-2127

2. Chapman PB, Hauschild A, Robert C, Haanen JB, Ascierto P, Larkin J, Dummer R, Garbe C, Testori A, Maio M, Hogg D, Lorigan P, Lebbe C, Jouary T, Schadendorf D, Ribas A, O'Day SJ, Sosman JA, Kirkwood JM, Eggermont AM, Dreno B, Nolop K, Li J, Nelson B, Hou J, Lee RJ, Flaherty KT, McArthur GA (2011) Improved survival with vemurafenib in melanoma with BRAF V600E mutation. N Engl J Med 364:2507-2516

3. Robert C, Thomas L, Bondarenko I, O'Day S, M DJ, Garbe C, Lebbe C, Baurain JF, Testori A, Grob JJ, Davidson N, Richards J, Maio M, Hauschild A, Miller WH Jr, Gascon P, Lotem M, Harmankaya K, Ibrahim R, Francis S, Chen TT, Humphrey R,
Hoos A, Wolchok JD (2011) Ipilimumab plus dacarbazine for previously untreated metastatic melanoma. N Engl J Med 364: $2517-2526$

4. Korn EL, Liu PY, Lee SJ, Chapman JA, Niedzwiecki D, Suman VJ, Moon J, Sondak VK, Atkins MB, Eisenhauer EA, Parulekar W, Markovic SN, Saxman S, Kirkwood JM (2008) Meta-analysis of phase II cooperative group trials in metastatic stage IV melanoma to determine progression-free and overall survival benchmarks for future phase II trials. J Clin Oncol 26:527-534

5. Boon T, Coulie PG, Van den Eynde B (1997) Tumor antigens recognized by $\mathrm{T}$ cells. Immunol Today 18:267-268

6. Rosenberg SA, Yang JC, Schwartzentruber DJ, Hwu P, Marincola FM, Topalian SL, Restifo NP, Dudley ME, Schwarz SL, Spiess PJ, Wunderlich JR, Parkhurst MR, Kawakami Y, Seipp CA, Einhorn JH, White DE (1998) Immunologic and therapeutic evaluation of a synthetic peptide vaccine for the treatment of patients with metastatic melanoma. Nat Med 4:321-327

7. Rosenberg SA, Yang JC, Restifo NP (2004) Cancer immunotherapy: moving beyond current vaccines. Nat Med 10:909-915

8. Ambrosini G, Adida C, Altieri DC (1997) A novel anti-apoptosis gene, survivin, expressed in cancer and lymphoma. Nat Med 3: 917-921

9. Islam A, Kageyama H, Takada N, Kawamoto T, Takayasu H, Isogai E, Ohira M, Hashizume K, Kobayashi H, Kaneko Y, Nakagawara A (2000) High expression of survivin, mapped to $17 \mathrm{q} 25$, is significantly associated with poor prognostic factors and promotes cell survival in human neuroblastoma. Oncogene 19: $617-623$

10. Kawasaki H, Altieri DC, Lu CD, Toyoda M, Tenjo T, Tanigawa N (1998) Inhibition of apoptosis by survivin predicts shorter survival rates in colorectal cancer. Cancer Res 58:5071-5074

11. Andersen MH, Svane IM, Becker JC, Straten PT (2007) The universal character of the tumor-associated antigen survivin. Clin Cancer Res 13:5991-5994

12. Cheever MA, Allison JP, Ferris AS, Finn OJ, Hastings BM, Hecht TT, Mellman I, Prindiville SA, Viner JL, Weiner LM, Matrisian LM (2009) The prioritization of cancer antigens: a National Cancer Institute pilot project for the acceleration of translational research. Clin Cancer Res 15:5323-5337

13. Xiang R, Mizutani N, Luo Y, Chiodoni C, Zhou H, Mizutani M, Ba Y, Becker JC, Reisfeld RA (2005) A DNA vaccine targeting survivin combines apoptosis with suppression of angiogenesis in lung tumor eradication. Cancer Res 65:553-561

14. Andersen MH, Becker JC, Straten P (2005) Regulators of apoptosis: suitable targets for immune therapy of cancer. Nat Rev Drug Discov 4:399-409

15. Otto K, Andersen MH, Eggert A, Keikavoussi P, Pedersen LO, Rath JC, Bock M, Brocker EB, Straten PT, Kampgen E, Becker JC (2005) Lack of toxicity of therapy-induced T cell responses against the universal tumour antigen survivin. Vaccine 23: 884-889

16. Wobser M, Keikavoussi P, Kunzmann V, Weininger M, Andersen MH, Becker JC (2006) Complete remission of liver metastasis of pancreatic cancer under vaccination with a HLA-A2 restricted peptide derived from the universal tumor antigen survivin. Cancer Immunol Immunother 55:1294-1298

17. Balch CM, Buzaid AC, Soong SJ, Atkins MB, Cascinelli N, Coit DG, Fleming ID, Gershenwald JE, Houghton A Jr, Kirkwood JM, McMasters KM, Mihm MF, Morton DL, Reintgen DS, Ross MI, Sober A, Thompson JA, Thompson JF (2001) Final version of the American Joint Committee on Cancer staging system for cutaneous melanoma. J Clin Oncol 19:3635-3648

18. Therasse P, Arbuck SG, Eisenhauer EA, Wanders J, Kaplan RS, Rubinstein L, Verweij J, Van Glabbeke M, van Oosterom AT, Christian MC, Gwyther SG (2000) New guidelines to evaluate the response to treatment in solid tumors. European Organization for 
Research and Treatment of Cancer, National Cancer Institute of the United States, National Cancer Institute of Canada. J Natl Cancer Inst 92:205-216

19. Andersen MH, Pedersen LO, Becker JC, Straten PT (2001) Identification of a cytotoxic $\mathrm{T}$ lymphocyte response to the apoptosis inhibitor protein survivin in cancer patients. Cancer Res 61:869-872

20. Andersen MH, Pedersen LO, Capeller B, Brocker EB, Becker JC, thor Straten P (2001) Spontaneous cytotoxic T-cell responses against survivin-derived MHC class I-restricted T-cell epitopes in situ as well as ex vivo in cancer patients. Cancer Res 61: 5964-5968

21. Scheibenbogen C, Lee KH, Mayer S, Stevanovic S, Moebius U, Herr W, Rammensee HG, Keilholz U (1997) A sensitive ELISPOT assay for detection of CD8 + T lymphocytes specific for HLA class I-binding peptide epitopes derived from influenza proteins in the blood of healthy donors and melanoma patients. Clin Cancer Res 3:221-226

22. Altman JD, Moss PA, Goulder PJ, Barouch DH, McHeyzerWilliams MG, Bell JI, McMichael AJ, Davis MM (1996) Phenotypic analysis of antigen-specific T lymphocytes. Science 274 : 94-96

23. Walter S, Bioley G, Buhring HJ, Koch S, Wernet D, Zippelius A, Pawelec G, Romero P, Stevanovic S, Rammensee HG, Gouttefangeas C (2005) High frequencies of functionally impaired cytokeratin 18-specific CD8+ $\mathrm{T}$ cells in healthy HLA-A2+ donors. Eur J Immunol 35:2876-2885

24. Brookmeyer R, Crowley J (1982) A confidence interval for the median survival time. Biometrics 38:29-41
25. Belli F, Testori A, Rivoltini L, Maio M, Andreola G, Sertoli MR, Gallino G, Piris A, Cattelan A, Lazzari I, Carrabba M, Scita G, Santantonio C, Pilla L, Tragni G, Lombardo C, Arienti F, Marchiano A, Queirolo P, Bertolini F, Cova A, Lamaj E, Ascani L, Camerini R, Corsi M, Cascinelli N, Lewis JJ, Srivastava P, Parmiani G (2002) Vaccination of metastatic melanoma patients with autologous tumor-derived heat shock protein gp96-peptide complexes: clinical and immunologic findings. J Clin Oncol 20:4169-4180

26. Bianchi I, Lleo A, Gershwin ME, Invernizzi P (2011) The $X$ chromosome and immune associated genes. J Autoimmun (Epub ahead of print)

27. Britten CM, Gouttefangeas C, Welters MJ, Pawelec G, Koch S, Ottensmeier C, Mander A, Walter S, Paschen A, Muller-Berghaus J, Haas I, Mackensen A, Kollgaard T, thor Straten P, Schmitt M, Giannopoulos K, Maier R, Veelken H, Bertinetti C, Konur A, Huber C, Stevanovic S, Wolfel T, van der Burg SH (2008) The CIMT-monitoring panel: a two-step approach to harmonize the enumeration of antigen-specific CD8+ T lymphocytes by structural and functional assays. Cancer Immunol Immunother 57:289-302

28. Hoos A, Britten CM, Huber C, O'Donnell-Tormey J (2011) A methodological framework to enhance the clinical success of cancer immunotherapy. Nat Biotechnol 29:867-870

29. Derre L, Ferber M, Touvrey C, Devevre E, Zoete V, Leimgruber A, Romero P, Michielin O, Levy F, Speiser DE (2007) A novel population of human melanoma-specific CD8 $\mathrm{T}$ cells recognizes Melan-AMART-1 immunodominant nonapeptide but not the corresponding decapeptide. J Immunol 179:7635-7645 